\title{
Learning Neighborhoods for Metric Learning
}

\author{
Jun Wang, Adam Woznica, and Alexandros Kalousis \\ AI Lab, Department of Computer Science, University of Geneva, Switzerland \\ Department of Business Informatics, University of Applied Sciences, \\ Western Switzerland \\ \{jun.wang, adam.woznica\}@unige.ch, \\ alexandros.kalousis@hesge.ch
}

\begin{abstract}
Metric learning methods have been shown to perform well on different learning tasks. Many of them rely on target neighborhood relationships that are computed in the original feature space and remain fixed throughout learning. As a result, the learned metric reflects the original neighborhood relations. We propose a novel formulation of the metric learning problem in which, in addition to the metric, the target neighborhood relations are also learned in a two-step iterative approach. The new formulation can be seen as a generalization of many existing metric learning methods. The formulation includes a target neighbor assignment rule that assigns different numbers of neighbors to instances according to their quality; 'high quality' instances get more neighbors. We experiment with two of its instantiations that correspond to the metric learning algorithms LMNN and MCML and compare it to other metric learning methods on a number of datasets. The experimental results show state-of-the-art performance and provide evidence that learning the neighborhood relations does improve predictive performance.
\end{abstract}

Keywords: Metric Learning, Neighborhood Learning.

\section{Introduction}

The choice of the appropriate distance metric plays an important role in distancebased algorithms such as $k$-NN and $k$-Means clustering. The Euclidean metric is often the metric of choice, however, it may easily decrease the performance of these algorithms since it relies on the simple assumption that all features are equally informative. Metric learning is an effective way to overcome this limitation by learning the importance of difference features exploiting prior knowledge that comes in different forms. The most well studied metric learning paradigm is that of learning the Mahalanobis metric with a steadily expanding literature over the last years 19133210189516 .

Metric learning for classification relies on two interrelated concepts, similarity and dissimilarity constraints, and the target neighborhood. The latter defines for any given instance the instances that should be its neighbors and it is specified using similarity and dissimilarity constraints. In the absence of any other prior knowledge the similarity and dissimilarity constraints are derived from the class

P. Flach et al. (Eds.): ECML PKDD 2012, Part I, LNCS 7523, pp. 223-236, 2012.

(C) Springer-Verlag Berlin Heidelberg 2012 
labels; instances of the same class should be similar and instances of different classes should be dissimilar.

The target neighborhood can be constructed in a global or local manner. With a global target neighborhood all constraints over all instance pairs are active; all instances of the same class should be similar and all instances from different classes should be dissimilar 193. These admittedly hard to achieve constraints can be relaxed with the incorporation of slack variables 132109 . With a local target neighborhood the satisfiability of the constraints is examined within a local neighborhood 4,17/10,18. For any given instance we only need to ensure that we satisfy the constraints that involve that instance and instances from its local neighborhood. The resulting problem is considerably less constrained than what we get with the global approach and easier to solve. However, the appropriate definition of the local target neighborhood becomes now a critical component of the metric learning algorithm since it determines which constraints will be considered in the learning process. [18] defines the local target neighborhood of an instance as its $k$, same-class, nearest neighbors, under the Euclidean metric in the original space. Goldberger et al. 4] initialize the target neighborhood for each instance to all same-class instances. The local neighborhood is encoded as a soft-max function of a linear projection matrix and changes as a result of the metric learning. With the exception of [4, all other approaches whether global or local establish a target neighborhood prior to learning and keep it fixed throughout the learning process. Thus the metric that will be learned from these fixed neighborhood relations is constrained by them and will be a reflection of them. However, these relations are not necessarily optimal with respect to the learning problem that one is addressing.

In this paper we propose a novel formulation of the metric learning problem that includes in the learning process the learning of the local target neighborhood relations. The formulation is based on the fact that many metric learning algorithms can be seen as directly maximizing the sum of some quality measure of the target neighbor relationships under an explicit parametrization of the target neighborhoods. We cast the process of learning the neighborhood as a linear programming problem with a totally unimodular constraint matrix [14. An integer 0-1 solution of the target neighbor relationship is guaranteed by the totally unimodular constraint matrix. The number of the target neighbors does not need to be fixed, the formulation allows the assignment of a different number of target neighbors for each learning instance according to the instance's quality. We propose a two-step iterative optimization algorithm that learns the target neighborhood relationships and the distance metric. The proposed neighborhood learning method can be coupled with standard metric learning methods to learn the distance metric, as long as these can be cast as instances of our formulation.

We experiment with two instantiations of our approach where the Large Margin Nearest Neighbor (LMNN) 18] and Maximally Collapsing Metric Learning (MCML) [3] algorithms are used to learn the metric; we dub the respective instantiations LN-LMNN and LN-MCML. We performed a series of experiments on a number of classification problems in order to determine whether learning 
the neighborhood relations improves over only learning the distance metric. The experimental results show that this is indeed the case. In addition, we also compared our method with other state-of-the-art metric learning methods and show that it improves over the current state-of-the-art performance.

The paper is organized as follows. In section 2, we discuss in more detail the related work. In Section 3 we present the optimization problem of the Learning Neighborhoods for Metric Learning algorithm (LNML) and in Section 4 we discuss the properties of LNML. In Section [5] we instantiate our neighborhood learning method on LMNN and MCML. In Section 6 we present the experimental results and we finally conclude with Section 7 .

\section{Related Work}

The early work of Xing et al., [19, learns a Mahalanobis distance metric for clustering that tries to minimize the sum of pairwise distances between similar instances while keeping the sum of dissimilar instance distances greater than a threshold. The similar and dissimilar pairs are determined on the basis of prior knowledge. Globerson \& Roweis, [3] introduced the Maximally Collapsing Metric Learning (MCML). MCML uses a stochastic nearest neighbor selection rule which selects the nearest neighbor $\mathbf{x}_{j}$ of an instance $\mathbf{x}_{i}$ under some probability distribution. It casts the metric learning problem as an optimization problem that tries to minimize the distance between two probability distributions, an ideal one and a data dependent one. In the ideal distribution the selection probability is always one for instances of the same class and zero for instances of different class, defining in that manner the similarity and dissimilarity constraints under the global target neighborhood approach. In the data dependent distribution the selection probability is given by a soft max function of a Mahalanobis distance metric, parametrized by the matrix $\mathbf{M}$ to be learned. In a similar spirit Davis et al., [2, introduced Information-Theoretic Metric Learning. ITML learns a Mahalanobis metric for classification with similarities and dissimilarities constraints that follow the global target neighborhood approach. In ITML all same-class instance pairs should have a distance smaller than some threshold and all different-class instance pairs should have a distance larger than some threshold. In addition the objective function of ITML seeks to minimize the distance between the learned metric matrix and a prior metric matrix, modelling like that prior knowledge about the metric if such is available. The optimization problem is cast as a distance of distributions subject to the pairwise constraints and finally expressed as a Bregman optimization problem (minimizing the LogDet divergence). In order to be able to find a feasible solution they introduce slack variables in the similarity and dissimilarity constraints.

The so far discussed metric learning methods follow the global target neighborhood approach in which all instances of the same class should be similar under the learned metric, and all pairs of instances from different classes dissimilar. This is a rather hard constraint and assumes that there is a linear projection of the original feature space that results in unimodal class conditional distributions. Goldberger et al., 4, proposed the NCA metric learning method which 
uses the same stochastic nearest neighbor selection rule under the same datadependent probability distribution as MCML. NCA seeks to minimize the soft error under its stochastic nearest neighbor selection rule. It uses only similarity constraints and the original target neighborhood of an instance is the set of all same-class instances. After metric learning some, but not necessarily all, same class instances will end up having high probability of been selecting as nearest neighbors of a given instance, thus having a small distance, while the others will be pushed further away. NCA thus learns the local target neighborhood as a part of the optimization. Nevertheless it is prone to overfitting, [20], and does not scale to large datasets. The large margin nearest neighbor method (LMNN) described in 17 18 learns a distance metric which directly minimizes the distances of each instance to its local target neighbors while keeping a large margin between them and different class instances. The target neighbors have to be specified prior to metric learning and in the absence of prior knowledge these are the $k$ same class nearest neighbors for each instance.

\section{Learning Target Neighborhoods for Metric Learning}

Given a set of training instances $\left\{\left(\mathbf{x}_{1}, y_{1}\right),\left(\mathbf{x}_{2}, y_{2}\right), \ldots,\left(\mathbf{x}_{n}, y_{n}\right)\right\}$ where $\mathbf{x}_{i} \in \mathbb{R}^{d}$ and the class labels $y_{i} \in\{1,2, \ldots, c\}$, the Mahalanobis distance between two instances $\mathbf{x}_{i}$ and $\mathbf{x}_{j}$ is defined as:

$$
D_{\mathbf{M}}\left(\mathbf{x}_{i}, \mathbf{x}_{j}\right)=\left(\mathbf{x}_{i}-\mathbf{x}_{j}\right)^{T} \mathbf{M}\left(\mathbf{x}_{i}-\mathbf{x}_{j}\right)
$$

where $\mathbf{M}$ is a Positive Semi-Definite (PSD) matrix $(\mathbf{M} \succeq 0)$ that we will learn.

We can reformulate many of the existing metric learning methods, such as [19 13 3 10 18, by explicitly parametrizing the target neighborhood relations as follows:

$$
\begin{aligned}
& \min _{\mathbf{M}, \boldsymbol{\Xi}} \sum_{i j, i \neq j, y_{i}=y_{j}} \mathbf{P}_{i j} \cdot F_{i j}(\mathbf{M}, \boldsymbol{\Xi}) \\
& \text { s.t. } \text { constraints of the original problem }
\end{aligned}
$$

The matrix $\mathbf{P}, \mathbf{P}_{i j} \in\{0,1\}$, describes the target neighbor relationships which are established prior to metric learning and are not altered in these methods. $\mathbf{P}_{i j}=1$, if $\mathbf{x}_{j}$ is the target neighbor of $\mathbf{x}_{i}$, otherwise, $\mathbf{P}_{i j}=0$. Note that the parameters $\mathbf{P}_{i i}$ and $\mathbf{P}_{i j}: y_{i} \neq y_{j}$ are set to zero, since an instance $\mathbf{x}_{i}$ cannot be a target neighbor of itself and the target neighbor relationship is constrained to same-class instances. This is why we have $i \neq j, y_{i}=y_{j}$ in the sum, however, for simplicity we will drop it from the following equations. $F_{i j}(\mathbf{M}, \boldsymbol{\Xi})$ is the term of the objective function of the metric learning methods that relates to the target neighbor relationship $\mathbf{P}_{i j}, \mathbf{M}$ is the Mahalanobis metric that we want to learn, and $\boldsymbol{\Xi}$ is a set of other parameters in the original metric learning problems, e.g. slack variables. Regularization terms on the $\mathbf{M}$ and $\boldsymbol{\Xi}$ parameters can also be added into Problem 21310.

The $F_{i j}(\mathbf{M}, \boldsymbol{\Xi})$ term can be seen as the 'quality' of the target neighbor relationship $\mathbf{P}_{i j}$ under the distance metric $\mathbf{M}$; a low value indicates a high quality 
neighbor relationship $\mathbf{P}_{i j}$. The different metric learning methods learn the $\mathbf{M}$ matrix that optimizes the sum of the quality terms based on the a priori established target neighbor relationships; however, there is no reason to believe that these target relationships are the most appropriate for learning.

To overcome the constraints imposed by the fixed target neighbors we propose the Learning the Neighborhood for Metric Learning method (LNML) in which, in addition to the metric matrix $\mathbf{M}$, we also learn the target neighborhood matrix P. LNML has as objective function the one given in Problem 2 which we now optimize also over the target neighborhood matrix $\mathbf{P}$. We add some new constraints in Problem 2 which control for the size of the target neighborhoods. The new optimization problem is the following:

$$
\begin{aligned}
\min _{\mathbf{M}, \Xi, \mathbf{P}} & \sum_{i j} \mathbf{P}_{i j} \cdot F_{i j}(\mathbf{M}, \Xi) \\
\text { s.t. } & \sum_{i, j} \mathbf{P}_{i j}=K_{a v} * n \\
& K_{\max } \geq \sum_{j} \mathbf{P}_{i, j} \geq K_{\min } \\
& 1 \geq \mathbf{P}_{i j} \geq 0 \\
& \text { constraints of the original problem }
\end{aligned}
$$

$K_{\min }$ and $K_{\max }$ are the minimum and maximum numbers of target neighbors that an instance can have. Thus the second constraint controls the number of target neighbor that $\mathbf{x}_{i}$ instance can have. $K_{a v}$ is the average number of target neighbor per instance. It holds by construction that $K_{\max } \geq K_{a v} \geq K_{\min }$. We should note here that we relax the target neighborhood matrix so that its elements $\mathbf{P}_{i j}$ take values in $[0,1]$ (third constraint). However, we will show later that a solution $\mathbf{P}_{i j} \in\{0,1\}$ is obtained, given some natural constraints on the $K_{\min }, K_{\max }$ and $K_{a v}$ parameters.

\subsection{Target Neighbor Assignment Rule}

Unlike other metric learning methods, e.g. LMNN, in which the number of target neighbors is fixed, LNML can assign a different number of target neighbors for each instance. As we saw the first constraint in Problem 3 sets the average number of target neighbors per instance to $K_{a v}$, while the second constraint limits the number of target neighbors for each instance between $K_{\min }$ and $K_{\max }$. The above optimization problem implements a target neighbor assignment rule which assigns more target neighbors to instances that have high quality target neighbor relations. We do so in order to avoid overfitting since most often the 'good' quality instances defined by metric learning algorithms 318] are instances in dense areas with low classification error. As a result the geometry of the dense areas of the different classes will be emphasized. How much emphasis we give on good quality instances depends on the actual values of $K_{\min }$ and $K_{\max }$. In the limit one can set the value of $K_{\min }$ to zero; nevertheless the risk with such 
a strategy is to focus heavily on dense and easy to learn regions of the data and ignore important boundary instances that are useful for learning.

\section{Optimization}

\subsection{Properties of the Optimization Problem}

We will now show that we get integer solutions for the $\mathbf{P}$ matrix by solving a linear programming problem and analyze the properties of Problem 3 .

Lemma 1. Given $\mathbf{M}, \boldsymbol{\Xi}$, and $K_{\max } \geq K_{a v} \geq K_{\min }$ then $\mathbf{P}_{i j} \in\{0,1\}$, if $K_{\min }$, $K_{\max }$ and $K_{a v}$ are integers.

Proof. Given $\mathbf{M}$ and $\boldsymbol{\Xi}, F_{i j}(\mathbf{M}, \boldsymbol{\Xi})$ becomes a constant. We denote by $\mathbf{p}$ the vectorization of the target neighborhood matrix $\mathbf{P}$ which excludes the diagonal elements and $\mathbf{P}_{i j}: y_{i} \neq y_{j}$, and by $\mathbf{f}$ the respective vectorized version of the $F_{i j}$ terms. Then we rewrite Problem 3 as:

$$
\begin{aligned}
\min _{\mathbf{p}} & \mathbf{p}^{T} \mathbf{f} \\
\text { s.t. } & \underbrace{}_{n} \underbrace{K_{\text {max }}, \cdots, K_{\max }}_{n}, K_{a v} * n)^{T} \geq \mathbf{A p} \geq \\
& \underbrace{(\underbrace{}_{\text {min }}, \cdots, K_{\text {min }}}, K_{a v} * n)^{T} \\
& 1 \geq \mathbf{p}_{i} \geq 0
\end{aligned}
$$

The first and second constraints of Problem 3 are reformulated as the first constraint in Problem 4. A is a $(n+1) \times\left(\sum_{c_{l}} n_{c_{l}}^{2}-n\right)$ constraint matrix, where $n_{c_{l}}$ is the number of instances in class $c_{l}$

$$
A=\left[\begin{array}{cccc}
1 & 0 & \cdots & 0 \\
0 & 1 & \cdots & 0 \\
\vdots & \vdots & \ddots & \vdots \\
0 & 0 & \cdots & 1 \\
1 & 1 & \cdots & 1
\end{array}\right]
$$

where $\mathbf{1}(\mathbf{0})$ is the vector of ones (zeros). Its elements depends on the its position in the matrix $\mathbf{A}$. In its $i$ th column, all $\mathbf{1}(\mathbf{0})$ vectors have $n_{i}-1$ elements, where $n_{i}$ is the number of instances of class $c_{j}$ with $c_{j}=y_{p_{i}}$. According to the sufficient condition for total unimodularity (Theorem 7.3 in [14]) the constraint matrix $\mathbf{A}$ is a totally unimodular matrix. Thus, the constraint matrix $\mathbf{B}=[\mathbf{I},-\mathbf{I}, \mathbf{A},-\mathbf{A}]^{T}$ in the following equivalent problem also is a totally unimodular matrix (pp.268 in [12]). 


$$
\begin{array}{ll}
\min _{\mathbf{p}} & \mathbf{p}^{T} \mathbf{f} \\
\text { s.t. } & \mathbf{B p} \leq \mathbf{e} \\
& e=(\underbrace{1, \cdots, 1}_{\sum_{c_{l}} n_{c_{l}}^{2}-n}, \underbrace{0, \cdots, 0}_{\sum_{c_{l}} n_{c_{l}}^{2}-n}, \underbrace{K_{\max }, \cdots, K_{\max }}_{n}, \\
& K_{a v} * n, \underbrace{-K_{\min }, \cdots,-K_{\min }}_{n},-K_{a v} * n)^{T}
\end{array}
$$

Since $\mathbf{e}$ is an integer vector, provided $K_{\min }, K_{\max }$, and $K_{a v}$, are integers, and the constraint matrix $\mathbf{B}$ is totally unimodular, the above linear programming problem will only have integer solutions (Theorem 19.1a in [12). Therefore, for the solution $\mathbf{p}$ it will hold that $\mathbf{p}_{i} \in\{0,1\}$ and consequently $\mathbf{P}_{i j} \in\{0,1\}$.

Although the constraints to control the size of the target neighborhood are convex, the objective function in Problem 3 is not jointly convex in $\mathbf{P}$ and $(\mathbf{M}, \boldsymbol{\Xi})$. However, as shown in Lemma 1, the binary solution of $\mathbf{P}$ can be obtained by a simple linear program if we fix $(\mathbf{M}, \boldsymbol{\Xi})$. Thus, Problem 3 is individually convex in $\mathbf{P}$ and $(\mathbf{M}, \boldsymbol{\Xi})$, if the original metric learning method is convex; this condition holds for all the methods that can be coupled with our neighborhood learning method [1913 3 10 18].

\subsection{Optimization Algorithm}

Based on Lemma 1 and the individual convexity property we propose a general and easy to implement iterative algorithm to solve Problem 3 . The details are given in Algorithm 1. At the first step of the $k$ th iteration we learn the binary target neighborhood matrix $\mathbf{P}^{(k)}$ under a fixed metric matrix $\mathbf{M}^{(k-1)}$ and $\boldsymbol{\Xi}^{(k-1)}$, learned in the $k-1$ th iteration, by solving the linear programming problem described in Lemma 1, At the second step of the iteration we learn the metric matrix $\mathbf{M}^{(k)}$ and $\boldsymbol{\Xi}^{(k)}$ with the target neighborhood matrix $\mathbf{P}^{(k)}$ using as the initial metric matrix the $\mathbf{M}^{(k-1)}$. The second step is simply the application of a standard metric learning algorithm in which we set the target neighborhood matrix to the learned $\mathbf{P}^{(k)}$ and the initial metric matrix to $\mathbf{M}^{(k-1)}$. The convergence of proposed algorithm is guaranteed if the original metric learning problem is convex [1]. In our experiment, it most often converges in 5-10 iterations.

\section{$5 \quad$ Instantiating LNML}

In this section we will show how we instantiate our neighborhood learning method with two standard metric learning methods, LMNN and MCML, other possible instantiations include the metric learning methods presented in 19 13 10. 


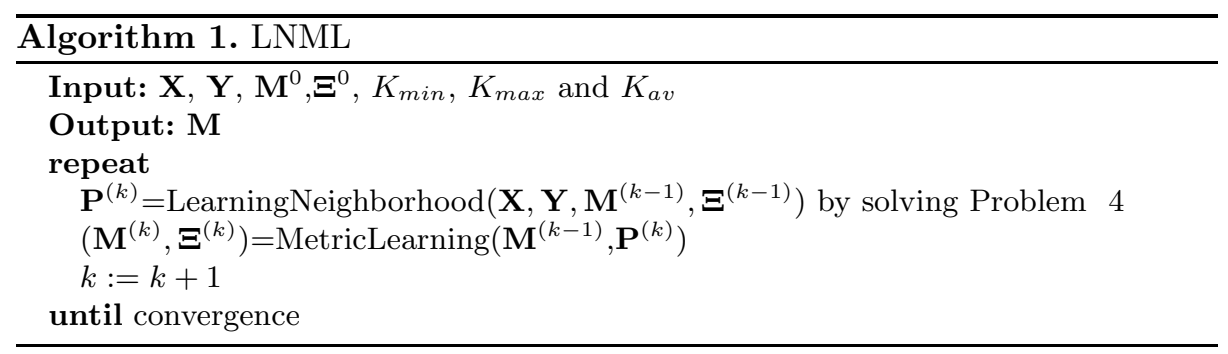

\subsection{Learning the Neighborhood for LMNN}

The optimization problem of LMNN is given by:

$$
\begin{aligned}
\min _{\mathbf{M}, \xi} & \sum_{i j} \mathbf{P}_{i j}\left\{(1-\mu) D_{\mathbf{M}}\left(\mathbf{x}_{i}, \mathbf{x}_{j}\right)+\mu \sum_{l}\left(1-\mathbf{Y}_{i l}\right) \xi_{i j l}\right\} \\
\text { s.t. } & D_{\mathbf{M}}\left(\mathbf{x}_{i}, \mathbf{x}_{l}\right)-D_{\mathbf{M}}\left(\mathbf{x}_{i}, \mathbf{x}_{j}\right) \geq 1-\xi_{i j l} \\
& \xi_{i j l}>0 \\
& \mathbf{M} \succeq 0
\end{aligned}
$$

where the matrix $\mathbf{Y}, \mathbf{Y}_{i j} \in\{0,1\}$, indicates whether the class labels $y_{i}$ and $y_{j}$ are the same $\left(\mathbf{Y}_{i j}=1\right)$ or different $\left(\mathbf{Y}_{i j}=0\right)$. The objective is to minimize the sum of the distances of all instances to their target neighbors while allowing for some errors, this trade off is controlled by the $\mu$ parameter. This is a convex optimization problem that has been shown to have good generalization ability and can be applied to large datasets. The original problem formulation corresponds to a fixed parametrization of $\mathbf{P}$ where its non-zero values are given by the $k$ nearest neighbors of the same class.

Coupling the neighborhood learning framework with the LMNN metric learning method results in the following optimization problem:

$$
\begin{aligned}
\min _{\mathbf{M}, \mathbf{P}, \xi} & \sum_{i j} \mathbf{P}_{i j} \cdot F_{i j}(\mathbf{M}, \xi) \\
=\min _{\mathbf{M}, \mathbf{P}, \xi} & \sum_{i j} \mathbf{P}_{i j}\left\{(1-\mu) D_{\mathbf{M}}\left(\mathbf{x}_{i}, \mathbf{x}_{j}\right)+\mu \sum_{l}\left(1-\mathbf{Y}_{i l}\right) \xi_{i j l}\right\} \\
\text { s.t. } & K_{\max } \geq \sum_{j} \mathbf{P}_{i, j} \geq K_{\min } \\
& \sum_{i, j} \mathbf{P}_{i j}=K_{a v} * n \\
& 1 \geq \mathbf{P}_{i j} \geq 0 \\
& D_{\mathbf{M}}\left(\mathbf{x}_{i}, \mathbf{x}_{l}\right)-D_{\mathbf{M}}\left(\mathbf{x}_{i}, \mathbf{x}_{j}\right) \geq 1-\xi_{i j l} \\
& \xi_{i j l}>0 \\
& \mathbf{M} \succeq 0
\end{aligned}
$$


We will call this coupling of LNML and LMNN LN-LMNN. The target neighbor assignment rule of LN-LMNN assigns more target neighbors to instances that have small distances from their target neighbors and low hinge loss.

\subsection{Learning the Neighborhood for MCML}

MCML relies on a data dependent stochastic probability that an instance $\mathbf{x}_{j}$ is selected as the nearest neighbor of an instance $\mathbf{x}_{i}$; this probability is given by:

$$
p_{\mathbf{M}}(j \mid i)=\frac{e^{-D_{\mathbf{M}}\left(\mathbf{x}_{i}, \mathbf{x}_{j}\right)}}{Z_{i}}=\frac{e^{-D_{\mathbf{M}}\left(\mathbf{x}_{i}, \mathbf{x}_{j}\right)}}{\sum_{k \neq i} e^{-D_{\mathbf{M}}\left(\mathbf{x}_{i}, \mathbf{x}_{k}\right)}}, i \neq j
$$

MCML learns the Mahalanobis metric that minimizes the KL divergence distance between this probability distribution and the ideal probability distribution $p_{0}$ given by:

$$
p_{0}(j \mid i)=\frac{\mathbf{P}_{i j}}{\sum_{k} \mathbf{P}_{i k}}, p_{0}(i \mid i)=0
$$

where $\mathbf{P}_{i j}=1$, if instance $\mathbf{x}_{j}$ is the target neighbor of instance $\mathbf{x}_{i}$, otherwise, $\mathbf{P}_{i j}=0$. The optimization problem of MCML is given by:

$$
\begin{aligned}
& \min _{\mathbf{M}} \sum_{i} K L\left[p_{0}(j \mid i) \mid p_{\mathbf{M}}(j \mid i)\right] \\
= & \min _{\mathbf{M}} \sum_{i, j} \mathbf{P}_{i j} \frac{\left(D_{\mathbf{M}}\left(\mathbf{x}_{i}, \mathbf{x}_{j}\right)+\log Z_{i}\right)}{\sum_{k} \mathbf{P}_{i k}} \\
\text { s.t. } \mathbf{M} \succeq 0 &
\end{aligned}
$$

Like LMNN, this is also a convex optimization problem. In the original problem formulation the ideal distribution is defined based on class labels, i.e. $\mathbf{P}_{i j}=1$, if instances $\mathbf{x}_{i}$ and $\mathbf{x}_{j}$ share the same class label, otherwise, $\mathbf{P}_{i j}=0$.

The neighborhood learning method cannot learn directly the target neighborhood for MCML, since the objective function of the latter cannot be rewritten in the form of the objective function in Problem [3, due to the denominator $\sum_{k} \mathbf{P}_{i k}$. However, if we fix the size of the neighborhood to $\sum_{k} \mathbf{P}_{i, k}=K_{a v}=$ $K_{\min }=K_{\max }$ the two methods can be coupled and the resulting optimization is given by:

$$
\begin{aligned}
& \min _{\mathbf{M}, \mathbf{P}} \sum_{i j} \mathbf{P}_{i j} \cdot F_{i j}(\mathbf{M}) \\
&= \min _{\mathbf{M}, \mathbf{P}} \sum_{i, j} \mathbf{P}_{i j} \frac{\left(D_{\mathbf{M}}\left(\mathbf{x}_{i}, \mathbf{x}_{j}\right)+\log Z_{i}\right)}{K_{a v}} \\
& \text { s.t. } \sum_{j} \mathbf{P}_{i, j}=K_{a v} \\
& \mathbf{M} \succeq 0
\end{aligned}
$$


We will dub this coupling of LNML and MCML as LN-MCML. The original MCML method follows the global approach in establishing the neighborhood, with LN-MCML we get a local approach in which the neighborhoods are of fixed size $K_{a v}$ for every instance.

\section{Experiments}

With the experiments we wish to investigate a number of issues. First, we want to examine whether learning the target neighborhood relations in the metric learning process can improve predictive performance over the baseline approach of metric learning with an apriori established target neighborhood. Second, we want to acquire an initial understanding of how the parameters $K_{\min }$ and $K_{\max }$ relate to the predictive performance. To this end, we will examine the predictive performance of LN-LMNN with two fold inner Cross Validation (CV) to select the appropriate values of $K_{\min }$ and $K_{\max }$, method which we will denote by LN-LMNN(CV), and that of LN-LMNN, with a default setting of $K_{\min }=K_{\max }=K_{a v}$. Finally, we want to see how the method that we propose compares to other state of the art metric learning methods, namely NCA and ITML. We include as an additional baseline in our experiments the performance of the Euclidean metric (EucMetric). We experimented with twelve different datasets: seven from the UCI machine learning repository, Sonar, Ionosphere, Iris, Balance, Wine, Letter, Isolet; four text mining datasets, Function, Alt, Disease and Structure, which were constructed from biological corpora [7; and MNIST 8], a handwritten digit recognition problem. A more detailed description of the datasets is given in Table 1 .

Since LMNN is computationally expensive for datasets with large number of features we applied principal component analysis (PCA) to retain a limited number of principal components, following [18. The datasets to which this was done were the four text mining datasets, Isolet and MNIST. For the two latter 173 and 164 principal components were respectively retained that explain $95 \%$ of the total variance. For the text mining datasets more than 1300 principal components should be retained to explain $95 \%$ of the total variance. Considering the running time constraints, we kept the 300 most important principal components which explained $52.45 \%, 47.57 \%, 44.30 \%$ and $48.16 \%$ of the total variance for respectively Alt, Disease, Function and Structure. We could experiment with NCA and MCML on full tranining datasets only with datasets with a small number of instances due to their computational complexity. For completeness we experimented with NCA on large datasets by undersampling the training instances, i.e. the learning process only involved $10 \%$ of full training instances which was the maximum number we could experiment for each dataset. We also applied ITML on both versions of the larger datasets, i.e. with PCA-based dimensionality reduction and the original ones.

For ITML, we randomly generate for each dataset the default $20 c^{2}$ constraints which are bounded repectively by the 5 th and 95 th percentiles of the distribution of all available pairwise distances for similar and dissimilar pairs. The slack 
Table 1. Datasets

\begin{tabular}{|c|c|c|c|c|c|c|}
\hline Datasets & Description & \# Sample\# & Feature\# & Class \# & Retained PCA Components\% & Explained Variance \\
\hline Sonar & & 208 & 60 & 2 & NA & NA \\
\hline Ionosphere & & 351 & 34 & 2 & NA & NA \\
\hline Wine & & 178 & 13 & 3 & NA & NA \\
\hline Balance & & 625 & 4 & 3 & NA & NA \\
\hline Letter & character recognition & 20000 & 16 & 26 & NA & $\mathrm{NA}$ \\
\hline Disease & sentence classification & 3273 & 2376 & 2 & 300 & $47.57 \%$ \\
\hline Structure & sentence classification & 3584 & 2368 & 2 & 300 & $48.16 \%$ \\
\hline Isolet & spoken character recognition & 7797 & 619 & 26 & 173 & $95 \%$ \\
\hline MNIST & handwritten digit recognition & 70000 & 784 & 26 & 164 & $95 \%$ \\
\hline
\end{tabular}

variable $\gamma$ is chosen form $\left\{10^{i}\right\}_{i=-4}^{4}$ using two-fold CV. The default identity matrix is employed as the regularization matrix. For the different instantiations of the LNML method we took care to have the same parameter settings for the encapsulated metric learning method and the respective baseline metric learning. For LN-LMNN, LN-LMNN(CV) and LMNN the regularization parameter $\mu$ that controls the trade-off between the distance minimization component and the hinge loss component was set to 0.5 (the default value of LMNN). For LMNN the default number of target neighbors was used (three). For LN-LMNN, we set $K_{\min }=K_{\max }=K_{a v}=3$, similar to LMNN. To explore the effect of a flexible neighborhood, the values of the $K_{\min }$ and $K_{\max }$ parameters in LN-LMNN(CV) were selected from the sets $\{1,4,3\}$ and $\{2,5,3\}$ respectively, while $K_{a v}$ was fixed to three. Similarly for LN-MCML we also set $K_{a v}=3$. The distance metrics for all methods are initialized to the Euclidean metric. As the classification algorithm we used 1-Nearest Neighbor.

We used 10-fold cross validation for all datasets to estimate classification accuracy, with the exception of Isolet and MNIST for which the default train and test split was used. The statistical significance of the differences were tested with McNemar's test and the p-value was set to 0.05 . In order to get a better understanding of the relative performance of the different algorithms for a given dataset we used a ranking schema in which an algorithm A was assigned one point if it was found to have a significantly better accuracy than another algorithm B, 0.5 points if the two algorithms did not have a significantly different performance, and zero points if A was found to be significantly worse than B. The rank of an algorithm for a given dataset is simply the sum of the points over the different pairwise comparisons. When comparing $N$ algorithms in a single dataset the highest possible score is $N-1$ while if there is no significant difference each algorithm will get $(N-1) / 2$ points.

\subsection{Results}

The results are presented in Table2, Examining whether learning also the neighborhood improves the predictive performance compared to plain metric learning, we see that in the case of LN-MCML, and for the five small datasets for which we have results, learning the neighborhood results in a statistically significant 
deterioration of the accuracy in one out of the five datasets (balance), while for the remaining four the differences were not statistically significant. If we now examine LN-LMNN(CV), LN-LMNN and LMNN we see that here learning the neighborhood does bring a statistically significant improvement. More precisely, LN-LMNN(CV) and LN-LMNN improve over LMNN respectively in six (two small and four large) and four (two small and two large) out of the 12 datasets. Moreove, by comparing LN-LMNN(CV) and LN-LMNN, we see that learning a flexible neighborhood with LN-LMNN $(\mathrm{CV})$ improves significantly the performance over LN-LMNN on two datasets. The low performance of LNMCML on the balance dataset was intriguing; in order to take a closer look we tried to determine automatically the appropriate target neighborhood size, $K_{a v}$, by selecting it on the basis of five-fold inner cross validation from the set $K_{a v}=\{3,5,7,10,20,30\}$. The results showed that the default value of $K_{a v}$ was too small for the given dataset, with the average selected size of the target neighborhood at 29. As a result of the automatic tunning of the target neighborhood size the predictive performance of LN-MCML jumped at an accuracy of $93.92 \%$ which represented a significant improvement over the baseline MCML for the balance dataset. For the remaining datasets it turned out that the choice of $K_{a v}=3$ was a good default choice. In any case, determining the appropriate size of the target neighborhood and how that affects the predictive performance is an issue that we wish to investigate further. In terms of the total score that the different methods obtain the $\mathrm{LN}-\mathrm{LMNN}(\mathrm{CV})$ achieves the best in both the

Table 2. Accuracy results. The superscripts ${ }^{+-=}$next to the LN-XXXX accuracy indicate the result of the McNemar's statistical test result of its comparison to the accuracy of XXXX and denote respectively a significant win, loss or no difference for LN-XXXX. Similarly, the superscripts ${ }^{+-}=$next to the LN-LMNN(CV) accuracy indicate the result of its comparison to the accuracies of LMNN and LN-LMNN. The bold entries for each dataset have no significant difference from the best accuracy for that dataset. The number in the parenthesis indicates the score of the respective algorithm for the given dataset based on the pairwise comparisons.

(a) Small datasets

\begin{tabular}{l|cc|ccc|c|c|c} 
Datasets & MCML & LN-MCML & LMNN & LN-LMNN & LN-LMNN $(C V)$ & EucMetric & NCA & ITML \\
\hline Sonar & $\mathbf{8 2 . 6 9}(3.5)$ & $\mathbf{8 4 . 6 2}(3.5)^{=}$ & $\mathbf{8 1 . 2 5}(3.5)$ & $\mathbf{8 1 . 2 5}(3.5)^{=}$ & $\mathbf{8 3 . 1 7}(3.5)^{=}=$ & $\mathbf{8 0 . 7 7}(3.5)$ & $\mathbf{8 1 . 7 3}(3.5)$ & $\mathbf{8 2 . 6 9}(3.5)$ \\
Ionosphere & $88.03(3.0)$ & $\mathbf{8 8 . 8 9}(3.5)^{=}$ & $\mathbf{8 9 . 1 7}(3.5)$ & $87.75(3.0)^{=}$ & $\mathbf{9 2 . 0 2}(5.5)^{=+}$ & $86.32(3.0)$ & $\mathbf{8 8 . 6 0}(3.5)$ & $87.75(3.0)$ \\
Wine & $91.57(3.0)$ & $\mathbf{9 6 . 0 7}(4.0)^{=}$ & $94.38(3.0)$ & $\mathbf{9 7 . 7 5}(5.5)^{+}$ & $\mathbf{9 7 . 7 5}(5.5)^{+=}$ & $76.97(0.0)$ & $91.57(3.0)$ & $\mathbf{9 4 . 9 4}(4.0)$ \\
Iris & $\mathbf{9 8 . 0 0}(4.5)$ & $\mathbf{9 6 . 0 0}(3.5)^{=}$ & $\mathbf{9 6 . 0 0}(3.5)$ & $94.00(3.0)^{=}$ & $94.00(3.0)^{=}=$ & $\mathbf{9 6 . 0 0}(3.5)$ & $\mathbf{9 6 . 0 0}(3.5)$ & $\mathbf{9 6 . 0 0}(3.5)$ \\
Balance & $91.20(5.0)$ & $78.08(1.0)^{-}$ & $78.56(1.0)$ & $89.12(4.5)^{+}$ & $89.28(4.5)^{+=}$ & $78.72(1.0)$ & $\mathbf{9 6 . 3 2}(7.0)$ & $87.84(4.0)$ \\
\hline Total Score & 19.0 & 15.5 & 14.5 & 19.5 & 22.0 & 11.0 & 20.5 & 18.0
\end{tabular}

(b) Large datasets

\begin{tabular}{l|ccc|ccc|c|c|c} 
Datasets & \multicolumn{2}{|c|}{ PCA+LMNN } & PCA+LN-LMNN PCA+LN-LMNN(CV) & EucMetric & PCA+EucMetric & PCA+NCA & ITML & PCA+ITML \\
\hline Letter & $96.86(5.0)$ & $\mathbf{9 7 . 7 1}(6.5)^{+}$ & $\mathbf{9 7 . 6 4}(6.5)^{+=}$ & $96.02(0.5)$ & $96.02(0.5)$ & $96.48(3.0)$ & $96.39(3.0)$ & $96.39(3.0)$ \\
Function & $76.30(2.5)$ & $76.73(2.5)^{=}$ & $\mathbf{7 8 . 9 1}(6.0)^{++}$ & $\mathbf{7 8 . 7 3}(6.0)$ & $76.48(2.5)$ & $72.36(0.0)$ & $\mathbf{7 8 . 7 3}(6.0)$ & $76.45(2.5)$ \\
Alt & $83.98(5.0)$ & $\mathbf{8 4 . 9 2}(6.5)^{+}$ & $\mathbf{8 5 . 3 7}(6.5)^{+=}$ & $68.51(0.5)$ & $71.33(2.0)$ & $78.54(4.0)$ & $68.49(0.5)$ & $72.53(3.0)$ \\
Disease & $\mathbf{8 0 . 2 3}(4.0)$ & $\mathbf{8 0 . 1 4}(4.0)^{=}$ & $\mathbf{8 0 . 6 6}(4.0)^{==}$ & $\mathbf{8 0 . 6 0}(4.0)$ & $\mathbf{8 0 . 2 3}(4.0)$ & $73.59(0.0)$ & $\mathbf{8 0 . 6 0}(4.0)$ & $\mathbf{8 0 . 1 4}(4.0)$ \\
Structure & $77.87(4.5)$ & $\mathbf{7 8 . 8 3}(6.0)^{=}$ & $\mathbf{7 9 . 3 7}(6.5)^{+=}$ & $75.82(1.5)$ & $77.00(4.0)$ & $71.93(0.0)$ & $75.79(1.5)$ & $77.06(4.0)$ \\
Isolet & $\mathbf{9 5 . 9 6}(6.0)$ & $\mathbf{9 5 . 0 6}(6.0)^{=}$ & $\mathbf{9 5 . 0 6}(6.0)^{==}$ & $88.58(1.5)$ & $88.33(1.5)$ & $85.63(0.0)$ & $92.05(3.5)$ & $91.08(3.5)$ \\
MNIST & $\mathbf{9 7 . 6 6}(6.0)$ & $\mathbf{9 7 . 6 6}(6.0)^{=}$ & $\mathbf{9 7 . 7 3}(6.0)^{==}$ & $96.91(2.0)$ & $96.97(2.0)$ & $96.58(1.5)$ & $96.93(1.5)$ & $97.09(3.0)$ \\
\hline Total Score & 33 & 37.5 & 41.5 & 16 & 16.5 & 8.5 & 20 & 23
\end{tabular}


small and large datasets. It is followed closely by NCA in the small datasets and by LN-LMNN in the large datasets.

\section{Conclusion and Future Work}

We presented LNML, a general Learning Neighborhood method for Metric Learning algorithms which couples the metric learning process with the process of establishing the appropriate target neighborhood for each instance, i.e. discovering for each instance which same class instances should be its neighbors. With the exception of NCA, which cannot be applied on datasets with many instances, all other metric learning methods whether they establish a global or a local target neighborhood do that prior to the metric learning and keep the target neighborhood fixed throughout the learning process. The metric that is learned as a result of the fixed neighborhoods simply reflects these original relations which are not necessarily optimal with respect to the classification problem that one is trying to solve. LNML lifts these constraints by learning the target neighborhood. We demonstrated it with two metric learning methods, LMNN and MCML. The experimental results show that learning the neighborhood can indeed improve the predictive performance.

The target neighborhood matrix $\mathbf{P}$ is strongly related to the similarity graphs which are often used in semi-supervised learning [6], spectral clustering [15] and manifold learning [11. Most often the similarity graphs in these methods are constructed in the original space, which nevertheless can be quite different from true manifold on which the data lies. These methods could also profit if one is able to learn the similarity graph instead of basing it on some prior structure.

Acknowledgments. This work was funded by the Swiss NSF (Grant 200021$122283 / 1)$. The support of the European Commission through EU projects DebugIT (FP7-217139) and e-LICO (FP7-231519) is also gratefully acknowledged.

\section{References}

1. Bezdek, J.C., Hathaway, R.J.: Some Notes on Alternating Optimization. In: Pal, N.R., Sugeno, M. (eds.) AFSS 2002. LNCS (LNAI), vol. 2275, pp. 288-300. Springer, Heidelberg (2002)

2. Davis, J.V., Kulis, B., Jain, P., Sra, S., Dhillon, I.S.: Information-theoretic metric learning. In: Proceedings of the 24th International Conference on Machine Learning. ACM, New York (2007)

3. Globerson, A., Roweis, S.: Metric learning by collapsing classes. In: Advances in Neural Information Processing Systems, vol. 18, MIT Press (2006)

4. Goldberger, J., Roweis, S., Hinton, G., Salakhutdinov, R.: Neighbourhood components analysis. In: Advances in Neural Information Processing Systems, vol. 17, MIT Press (2005)

5. Guillaumin, M., Verbeek, J., Schmid, C.: Is that you? Metric learning approaches for face identification. In: Proceedings of 12th International Conference on Computer Vision, pp. 498-505 (2009) 
6. Jebara, T., Wang, J., Chang, S.-F.: Graph construction and b-matching for semisupervised learning. In: Proceedings of the 26th Annual International Conference on Machine Learning, pp. 441-448. ACM, New York (2009)

7. Kalousis, A., Prados, J., Hilario, M.: Stability of feature selection algorithms: a study on high-dimensional spaces. Knowledge and Information Systems 12(1), 95$116(2007)$

8. LeCun, Y., Bottou, L., Bengio, Y., Haffner, P.: Gradient-based learning applied to document recognition. Proceedings of the IEEE 86, 2278-2324 (1998)

9. Lu, Z., Jain, P., Dhillon, I.S.: Geometry-aware metric learning. In: Proceedings of the 26th Annual International Conference on Machine Learning. ACM Press, New York (2009)

10. Nguyen, N., Guo, Y.: Metric Learning: A Support Vector Approach. In: Daelemans, W., Goethals, B., Morik, K. (eds.) ECML PKDD 2008, Part II. LNCS (LNAI), vol. 5212, pp. 125-136. Springer, Heidelberg (2008)

11. Roweis, S., Saul, L.: Nonlinear dimensionality reduction by locally linear embedding. Science 22, 2323-2326 (2000)

12. Schrijver, A.: Theory of linear and integer programming. John Wiley \& Sons Inc. (1998)

13. Schultz, M., Joachims, T.: Learning a distance metric from relative comparisons. In: Advances in Neural Information Processing Systems 16: Proceedings of the 2003 Conference, p. 41. MIT Press (2004)

14. Sierksma, G.: Linear and integer programming: theory and practice. CRC (2002)

15. von Luxburg, U.: A tutorial on spectral clustering. Statistics and Computing 17, 395-416 (2007)

16. Wang, J., Do, H., Woznica, A., Kalousis, A.: Metric learning with multiple kernels. In: Advances in Neural Information Processing Systems. MIT Press (2011)

17. Weinberger, K., Blitzer, J., Saul, L.: Distance metric learning for large margin nearest neighbor classification. In: Advances in Neural Information Processing Systems, vol. 18, MIT Press (2006)

18. Weinberger, K.Q., Saul, L.K.: Distance metric learning for large margin nearest neighbor classification. The Journal of Machine Learning Research 10, 207-244 (2009)

19. Xing, E.P., Ng, A.Y., Jordan, M.I., Russell, S.: Distance metric learning with application to clustering with side-information. In: Advances in Neural Information Processing Systems. MIT Press (2003)

20. Yang, Z., Laaksonen, J.: Regularized Neighborhood Component Analysis. In: Ersbøll, B.K., Pedersen, K.S. (eds.) SCIA 2007. LNCS, vol. 4522, pp. 253-262. Springer, Heidelberg (2007) 